\title{
On the Tate-Shafarevich group of semistable elliptic curves with a rational 3-torsion
}

\author{
by \\ Noboru Aoki (Tokyo)
}

1. Introduction. Let $E$ be an elliptic curve defined over the rational number field $\mathbb{Q}$ and $E(\mathbb{Q})$ the Mordell-Weil group of $\mathbb{Q}$-rational points on $E$. Let $n$ be an integer greater than one and $E_{n}$ the group of $n$-torsion points on $E$. The $n$-Selmer group $\operatorname{Sel}^{(n)}(E / \mathbb{Q})$ of $E / \mathbb{Q}$ is defined to be the kernel of the composite map

$$
H^{1}\left(\mathbb{Q}, E_{n}\right) \rightarrow \prod_{p} H^{1}\left(\mathbb{Q}_{p}, E_{n}\right) \rightarrow \prod_{p} H^{1}\left(\mathbb{Q}_{p}, E\right),
$$

where the first map is the direct product of restriction maps for all places $p$ of $\mathbb{Q}$ and the second map is the one induced from the inclusion $E_{n} \hookrightarrow E$. Then $\operatorname{Sel}^{(n)}(E / \mathbb{Q})$ is known to be finite for any $n$, and there is an injection from the quotient group $E(\mathbb{Q}) / n E(\mathbb{Q})$ into $\operatorname{Sel}^{(n)}(E / \mathbb{Q})$. Thus $\operatorname{Sel}^{(n)}(E / \mathbb{Q})$ gives an upper bound for the rank of $E(\mathbb{Q})$. Therefore, if $\operatorname{rank}(E(\mathbb{Q}))$ is unbounded when $E$ varies over the elliptic curves over $\mathbb{Q}$, then the order of $\operatorname{Sel}^{(n)}(E / \mathbb{Q})$ with $n$ fixed can be arbitrarily large. The converse, however, is not necessarily true because of the presence of the Tate-Shafarevich group

$$
\amalg(E / \mathbb{Q})=\operatorname{Ker}\left(H^{1}(\mathbb{Q}, E) \rightarrow \prod_{p} H^{1}\left(\mathbb{Q}_{p}, E\right)\right) .
$$

The $n$-torsion subgroup $\amalg(E / \mathbb{Q})_{n}$ of $\amalg(E / \mathbb{Q})$ fits into the exact sequence

$$
0 \rightarrow E(\mathbb{Q}) / n E(\mathbb{Q}) \rightarrow \operatorname{Sel}^{(n)}(E / \mathbb{Q}) \rightarrow \amalg(E / \mathbb{Q})_{n} \rightarrow 0 .
$$

Thus we are naturally led to the following problem: Given a prime number $n$ and a family $\mathscr{E}$ of elliptic curves over $\mathbb{Q}$, determine whether

$$
\sup \left\{\#\left(\amalg(E / \mathbb{Q})_{n}\right) \mid E \in \mathscr{E}\right\}=\infty
$$

or not. This problem has been studied for $n=2$ by Bölling [3], Kramer [8], Lemmermeyer [9] and Atake [1], for $n=3$ by Cassels [6], and for $n=5$ by Fisher [7]. The families of elliptic curves considered in those works may

2000 Mathematics Subject Classification: 11G05, 11G07, 14H52. 
be divided into two types: one is the family of (quadratic ([3], [9], [1]) or cubic $([6]))$ twists of a fixed elliptic curve, and the other is a one-parameter family of semistable elliptic curves with non-constant $j$-invariant ([8], [7]).

In this paper we will be mainly interested in two types of elliptic curves:

$$
\begin{aligned}
& E=E_{(a, b)}: \quad y^{2}+a x y+b y=x^{3}, \\
& F=F_{(a, b)}: \quad y^{2}+a x y+b y=x^{3}-5 a b x-a^{3} b-7 b^{2},
\end{aligned}
$$

where $a, b$ are relatively prime non-zero integers such that $a^{3}-27 b \neq 0$. One can easily see that $E$ has a rational point $S=(0,0) \in E(\mathbb{Q})$ of order 3 , and $F$ is the quotient of $E$ by the cyclic subgroup $\langle S\rangle$ generated by $S$. We consider the problem above for $n=3$ and the family of such elliptic curves $F_{a, b}$. We should remark that the assumption on $a$ and $b$ ensures that $E$ and $F$ are semistable elliptic curves, and so CM elliptic curves are excluded from our family in contrast to the work of Cassels mentioned above, where he treated the CM elliptic curves $x^{3}+y^{3}+d z^{3}=0$. The purpose of this paper is to prove the following theorem.

THEOREM 1.1. Let $\mathscr{E}$ be the set of elliptic curves $F_{(a, b)}$ defined above. Then

$$
\sup \left\{\#\left(H(F / \mathbb{Q})_{3}\right) \mid F \in \mathscr{E}\right\}=\infty .
$$

In the proof of Theorem 1.1 we will assume that $a^{3}-27 b$ is a prime number and $b$ is not a cube in $\mathbb{Q}$, hence neither $E_{3}$ nor $F_{3}$ splits over $\mathbb{Q}$. (Note that the discriminants of our curves are given by $\Delta_{E}=\left(a^{3}-27 b\right) b^{3}$ and $\Delta_{F}=\left(a^{3}-27 b\right)^{3} b$.) Therefore we cannot use the method of [4] and [7] to prove Theorem 1.1. We will instead compute a restriction of the Cassels-Tate pairing to a subgroup of $\amalg\left(F / \mathbb{Q}\left(E_{3}\right)\right)$ using McCallum's formula (see Theorem 6.5). This part was strongly influenced by the recent work of Beaver [2] and Fisher [7].

2. The Selmer group and the Tate-Shafarevich group. Let $n$ be a positive integer greater than one. Let $E$ be an elliptic curve defined over a number field $k$. Suppose $E(k)$ contains a point $S$ of order $n$ and let $F=E /\langle S\rangle$ be the quotient of $E$ by the cyclic group generated by $S$. Then $F$ is also defined over $k$ and the natural surjection $\varphi: E \rightarrow F$ is a ( $k$-rational) cyclic $n$-isogeny such that $E_{\varphi}:=\operatorname{Ker}(\varphi)=\langle S\rangle$. Since $S$ is rational over $k$, we have $E_{\varphi} \cong \mathbb{Z} / n \mathbb{Z}$ as $\operatorname{Gal}(\bar{k} / k)$-modules. Let $\psi: F \rightarrow E$ be the dual isogeny of $\varphi$. Then $F_{\psi}:=\operatorname{Ker}(\psi)$ is isomorphic to $\mu_{n}$ as a $\operatorname{Gal}(\bar{k} / k)$-module.

Now, let $L$ be a field containing $k$ and consider the exact sequence

$$
0 \rightarrow F_{\psi} \rightarrow F \stackrel{\psi}{\rightarrow} E \rightarrow 0
$$

of $\operatorname{Gal}(\bar{L} / L)$-modules. Taking Galois cohomology, we obtain the exact sequence 


$$
0 \rightarrow E(L) / \psi(F(L)) \stackrel{\delta_{L}^{(\psi)}}{\longrightarrow} H^{1}\left(L, F_{\psi}\right) \rightarrow H^{1}(L, F)_{\psi} \rightarrow 0 .
$$

Let $M_{k}$ be the set of places of $k$. For each $v \in M_{k}$, we denote by $k_{v}$ the completion of $k$ at $v$. Taking $k_{v}$ for $L$, we then obtain the exact sequence

$$
0 \rightarrow E\left(k_{v}\right) / \psi\left(F\left(k_{v}\right)\right) \stackrel{\delta_{v}^{(\psi)}}{\longrightarrow} H^{1}\left(k_{v}, F_{\psi}\right) \rightarrow H^{1}\left(k_{v}, F\right)_{\psi} \rightarrow 0,
$$

where $\delta_{v}^{(\psi)}=\delta_{k_{v}}^{(\psi)}$. Let $\operatorname{res}_{v}: H^{1}(k, *) \rightarrow H^{1}\left(k_{v}, *\right)$ denote the restriction map. We define the $\psi$-Selmer group by

$$
\begin{aligned}
\operatorname{Sel}^{(\psi)}(F / k) & =\operatorname{Ker}\left(H^{1}\left(k, F_{\psi}\right) \stackrel{\prod \operatorname{res}_{v}}{\longrightarrow} \prod_{v \in M_{k}} H^{1}\left(k_{v}, F_{\psi}\right) \rightarrow \prod_{v \in M_{k}} H^{1}\left(k_{v}, F\right)\right) \\
& =\left\{x \in H^{1}\left(k, F_{\psi}\right) \mid \operatorname{res}_{v}(x) \in \operatorname{Im}\left(\delta_{v}^{(\psi)}\right) \text { for all } v \in M_{k}\right\} .
\end{aligned}
$$

Since $F_{\psi} \cong \mu_{n}$, Kummer theory implies that $H^{1}\left(k, F_{\psi}\right) \cong k^{\times} / k^{\times n}$. In what follows we will identify $H^{1}\left(k, F_{\psi}\right)$ with $k^{\times} / k^{\times n}$ by this isomorphism. Thus $\operatorname{Sel}^{(\psi)}(F / k)$ may be viewed as a subgroup of $k^{\times} / k^{\times n}$. The following proposition will be useful when we give an explicit description of $\operatorname{Im}\left(\delta_{k}^{(\psi)}\right)$.

Proposition 2.1. There exists a rational function $f \in k(E)^{\times}$such that

$$
\operatorname{div}(f)=n((S)-(O)) \quad \text { and } \quad f \circ[n] \in\left(k(E)^{\times}\right)^{n},
$$

where $[n]$ denotes the multiplication-by-n map. Then

$$
\delta_{k}^{(\psi)}(P) \equiv f(P)\left(\bmod k^{\times n}\right)
$$

for any $P \in E(k) \backslash\{O, S\}$.

Proof. See [13, Chapter X, Theorem 1.1].

Define the Tate-Shafarevich group of $F / k$ by

$$
\amalg(F / k)=\operatorname{Ker}\left(H^{1}(k, F) \rightarrow \prod_{v \in M_{k}} H^{1}\left(k_{v}, F\right)\right) .
$$

It is conjectured that $\amalg(F / k)$ is finite. Let

$$
\langle,\rangle: \amalg(F / k) \times \amalg(F / k) \rightarrow \mathbb{Q} / \mathbb{Z}
$$

be the Cassels-Tate pairing on $\amalg(F / k)$. (See [5], [15] or [11] for the definition.) It is well known that this pairing is non-degenerate if and only if the divisible part of $\amalg(F / k)$ is trivial. Let $\amalg(F / k)_{\psi}$ be the kernel of the map $\amalg(F / k) \rightarrow \amalg(E / k)$ induced from $\psi$, and let

$$
\langle,\rangle_{\psi}: \amalg(F / k)_{\psi} \times \amalg(F / k)_{\psi} \rightarrow \frac{1}{n} \mathbb{Z} / \mathbb{Z} \cong \mathbb{Z} / n \mathbb{Z}
$$


be the restriction of $\langle$,$\rangle to the subgroup \amalg(F / k)_{\psi}$. The group $\amalg(F / k)_{\psi}$ fits into the exact sequence

$$
0 \rightarrow E(k) / \psi(F(k)) \stackrel{\delta_{k}^{(\psi)}}{\longrightarrow} \operatorname{Sel}^{(\psi)}(F / k) \rightarrow \amalg(F / k)_{\psi} \rightarrow 0 .
$$

Pulling back the pairing to $\operatorname{Sel}^{(\psi)}(F / k)$ using the surjection $\operatorname{Sel}^{(\psi)}(F / k) \rightarrow$ $\amalg(F / k)_{\psi}$, we obtain a pairing on $\operatorname{Sel}^{(\psi)}(F / k)$, which we denote by the same symbols:

$$
\langle,\rangle_{\psi}: \operatorname{Sel}^{(\psi)}(F / k) \times \operatorname{Sel}^{(\psi)}(F / k) \rightarrow \mathbb{Z} / n \mathbb{Z} .
$$

In Section 6 we will prove an explicit formula for the pairing $\langle,\rangle_{\psi}$ when $E$ is a semistable elliptic curve satisfying a certain condition on the discriminant of $E$.

3. Tate curves. Let $p$ be a prime number. Throughout this section $k$ will denote a $p$-adic field, that is, a finite extension of $\mathbb{Q}_{p}$. Let $v$ denote the valuation of $k$ such that $v\left(k^{\times}\right)=\mathbb{Z}$ and $q$ a non-zero element of $k$ with $v(q)>0$. Let $E=E_{q}$ be the Tate curve over $k$ defined by the equation

$$
y^{2}+x y=x^{3}+a_{4}(q) x+a_{6}(q),
$$

where $a_{4}(q)$ and $a_{6}(q)$ are convergent power series in $k[[u]]$ defined by

$$
a_{4}=-\sum_{n=1}^{\infty} \frac{n^{4} q^{n}}{1-q^{n}}, \quad a_{6}=-\frac{1}{12}\left(5 \sum_{n=1}^{\infty} \frac{n^{3} q^{n}}{1-q^{n}}+7 \sum_{n=1}^{\infty} \frac{n^{5} q^{n}}{1-q^{n}}\right) .
$$

(For more details on the Tate curve see [14, Chapter V].) Then we have an isomorphism of $\operatorname{Gal}(\bar{k} / k)$-modules called the Tate parametrization:

$$
\tau: \bar{k}^{\times} / q^{\mathbb{Z}} \rightarrow E(\bar{k}), \quad u \mapsto(X(u), Y(u)),
$$

where $X(u)$ and $Y(u)$ are convergent power series in $k[[u]]$ defined by

$$
\begin{aligned}
& X(u)=\frac{u}{(1-u)^{2}}+\sum_{n=1}^{\infty}\left(\frac{q^{n} u}{\left(1-q^{n} u\right)^{2}}+\frac{q^{n} u^{-1}}{\left(1-q^{n} u^{-1}\right)^{2}}-2 \frac{q^{n}}{\left(1-q^{n}\right)^{2}}\right) \\
& Y(u)=\frac{u^{2}}{(1-u)^{3}}+\sum_{n=1}^{\infty}\left(\frac{\left(q^{n} u\right)^{2}}{\left(1-q^{n} u\right)^{3}}-\frac{q^{n} u^{-1}}{\left(1-q^{n} u^{-1}\right)^{3}}+\frac{q^{n}}{\left(1-q^{n}\right)^{2}}\right) .
\end{aligned}
$$

Let $n$ be a prime number, and fix an $n$th root of unity $\zeta \in \mu_{n}$ and an $n$th root $q_{1}=q^{1 / n}$ of $q$ in $\bar{k}$. Then for any $P \in E_{n}$, we define two elements $\mu(P)$ and $\nu(P)$ of $\mathbb{Z} / n \mathbb{Z}$ by

$$
\tau\left(\zeta^{\mu(P)} q_{1}^{\nu(P)}\right)=P .
$$

Clearly both $\mu$ and $\nu$ are homomorphisms from $E_{n}$ to $\mathbb{Z} / n \mathbb{Z}$.

Now, let $S$ be a $k$-rational point of $E$ of order $n$. As in the preceding section we consider the quotient $F$ of $E$ by the cyclic subgroup generated by $S$ and the cyclic isogeny $\psi: F \rightarrow E$. 
Proposition 3.1. Let $\delta_{k}^{(\psi)}: E(k) \rightarrow H^{1}\left(k, F_{\psi}\right)=k^{\times} / k^{\times n}$ be the map defined in (1). Then

$$
\operatorname{Im}\left(\delta_{k}^{(\psi)}\right)= \begin{cases}k^{\times} / k^{\times n} & \text { if } \nu(S) \neq 0 \\ \{1\} & \text { if } \nu(S)=0 .\end{cases}
$$

This fact is well known; for example it is proved in [2] in the case of $n=5$ and the proof works for any $n$. However, we will give another proof using an explicit description of the rational function $f$ defined in Proposition 2.1. This proof is a generalization of that of Brumer and Kramer [4], where the case $n=2$ is treated. We consider the following theta function:

$$
\theta(u)=(1-u) \prod_{n=1}^{\infty} \frac{\left(1-q^{n} u\right)\left(1-q^{n} u^{-1}\right)}{\left(1-q^{n}\right)^{2}} \quad\left(u \in \bar{k}^{\times}\right) .
$$

Lemma 3.2. Let $x_{1}, \ldots, x_{r} \in \bar{k}^{\times}$and $m_{0}, m_{1}, \ldots, m_{r} \in \mathbb{Z}$. Let $f$ be a function on $\bar{k}^{\times}$defined by

$$
f(u)=u^{-m_{0}} \prod_{i=1}^{r} \theta\left(u / x_{i}\right)^{m_{i}} \quad\left(u \in \bar{k}^{\times}\right) .
$$

Then the equation $f(q u)=f(u)$ holds for all $u \in \bar{k}^{\times}$if and only if the following two conditions are satisfied:

$$
\sum_{i=1}^{r} m_{i}=0 \quad \text { and } \quad \prod_{i=1}^{r} x_{i}^{m_{i}}=q^{m_{0}} .
$$

Moreover, if these conditions are satisfied (hence $f \circ \tau^{-1}$ may be viewed as a rational function on the Tate curve $E$ ), then the divisor of the rational function $f$ on $E$ is given by

$$
\operatorname{div}\left(f \circ \tau^{-1}\right)=\sum_{i=1}^{r} m_{i}\left(\tau\left(x_{i}\right)\right) .
$$

Proof. See [12, $\S 1$, Proposition 1].

Proof of Proposition 3.1. We want to construct a rational function $f$ on $E$ which satisfies the condition of Proposition 2.1. Let $\mu=\mu(S), \nu=\nu(S)$ and define a function $f$ on $E$ by

$$
f(\tau(u))=u^{-\nu}\left(\frac{\theta\left(\zeta^{-\mu} q_{1}^{-\nu} u\right)}{\theta(u)}\right)^{n} \quad\left(u \in \bar{k}^{\times}\right) .
$$

Then Lemma 3.2 implies that $f$ is a rational function on $E$ defined over $k$ such that $\operatorname{div}(f)=n((S)-(O))$. Moreover, we define a function $g$ on $E$ by

$$
g(\tau(u))=u^{-\nu} \frac{\theta\left(\zeta^{-\mu} q_{1}^{-\nu} u^{n}\right)}{\theta\left(u^{n}\right)} .
$$


Then $g$ is also a rational function on $E$ defined over $k$ and satisfies the relation

$$
f\left(\tau\left(u^{n}\right)\right)=g(\tau(u))^{n} .
$$

Therefore $f \circ[n]=g^{n}$, so $f$ satisfies the condition in Proposition 2.1. Hence

$$
\delta_{k}^{(\psi)}(\tau(u)) \equiv f(\tau(u)) \equiv u^{-\nu}\left(\bmod k^{\times n}\right)
$$

for all $u \in k^{\times}$. Proposition 3.1 now easily follows from (7).

In the next proposition we identify $\mathbb{Z} / n \mathbb{Z}$ with the subset $\{0,1, \ldots, n-1\}$ of $\mathbb{Z}$. Thus we regard $\nu(P)$ as an integer such that $0 \leq \nu(P)<n$.

Proposition 3.3. Suppose $q_{1} \in k$. Let $f \in k(E)^{\times}$be the rational function on $E$ defined by (6). Then for any $P \in E(k)_{n} \backslash\{O, S\}, v(f(P))$ is given by the formula

$v(f(P))=-(\nu(S) \nu(P)-n \max \{\nu(S)-\nu(P), 0\}) v\left(q_{1}\right)-\delta_{\nu(S), \nu(P)} v(1-\zeta)$, where $\delta_{*, *}$ denotes Kronecker's delta.

Proof. For any $\alpha, \beta \in \bar{k}^{\times}$, we write $\alpha \sim \beta$ if $v(\alpha / \beta)=0$. Take $u, z \in \bar{k}^{\times}$ such that $\tau(u)=S, \tau(z)=P$. Clearly one can take $u, z$ so that $0 \leq$ $v(u), v(z)<v(q)$. Then by (6) we have

$$
f(P)=z^{-\nu(S)}\left(\frac{\theta\left(u^{-1} z\right)}{\theta(z)}\right)^{n} .
$$

Since $v(z)=\nu(P) v\left(q_{1}\right)$, this shows that

$$
v(f(P))=-\nu(S) \nu(P) v\left(q_{1}\right)+n \cdot v\left(\frac{\theta\left(u^{-1} z\right)}{\theta(z)}\right) .
$$

To calculate the second term, notice that $-v(q)<v(z / u)<v(q)$ and $0 \leq$ $v(z)<v(q)$. Hence $1-q^{n}(z / u)^{ \pm 1} \sim 1$ and $1-q^{n} z^{ \pm 1} \sim 1$ for all $n \geq 1$. Thus

$$
\frac{\theta\left(u^{-1} z\right)}{\theta(z)} \sim \frac{1-u^{-1} z}{1-z} .
$$

First, suppose $\nu(P) \neq 0$. Then $1-z \sim 1$ and

$$
1-u^{-1} z \sim \begin{cases}1 & \text { if } \nu(P)>\nu(S), \\ u^{-1} z & \text { if } \nu(P)<\nu(S), \\ 1-\zeta & \text { if } \nu(P)=\nu(S) .\end{cases}
$$

Here we have used the fact that $1-\zeta^{s} \sim 1-\zeta$ for any $0<s<n$. Therefore,

$$
v\left(\frac{\theta\left(u^{-1} z\right)}{\theta(z)}\right)=-\max \{\nu(S)-\nu(P), 0\} v\left(q_{1}\right)+\delta_{\nu(S), \nu(P)} v(1-\zeta) .
$$

From (8) and (9) we obtain the desired formula. 
Next, suppose $\nu(P)=0$. Then $\mu(P) \neq 0$, hence $1-z \sim 1-\zeta^{\mu(P)} \sim 1-\zeta$. Moreover, if $\nu(P)=0$, then $\mu(S) \neq \mu(P)$, and

$$
1-u^{-1} z \sim \begin{cases}u^{-1} & \text { if } \nu(S) \neq 0 \\ 1-\zeta & \text { if } \nu(S)=0 .\end{cases}
$$

Therefore,

$$
v\left(\frac{\theta\left(u^{-1} z\right)}{\theta(z)}\right)=-\max \{\nu(S), 0\} v\left(q_{1}\right)+\delta_{\nu(S), 0} v(1-\zeta) .
$$

From (8) and (10), we find that the formula of the proposition also holds in this case. This completes the proof.

Corollary 3.4. Suppose $n$ is a prime and $q_{1} \in k$. If $v(n)=0$, then $v(f(P))$ is divisible by $v\left(q_{1}\right)$ and the integer $v(f(P)) / v\left(q_{1}\right)$ satisfies the congruence

$$
\frac{v(f(P))}{v\left(q_{1}\right)} \equiv-\nu(S) \nu(P)(\bmod n) .
$$

Further, if $v(n)>0$ and $v\left(q_{1}\right) \not \equiv 0(\bmod n)\left(\right.$ hence $v\left(q_{1}\right)$ is an $n$-adic unit), then the same congruence holds.

Proof. If $v(n)=0$, then Proposition 3.3 implies that

$$
v(f(P))=-[\nu(S) \nu(P)+n \cdot \max \{\nu(S)-\nu(P), 0\}] v\left(q_{1}\right) .
$$

Hence the assertion of the proposition holds. If $v(n)>0$ and $v\left(q_{1}\right) \not \equiv 0$ $(\bmod n)$, then $v\left(q_{1}\right)$ is an $n$-adic unit, hence we get the congruence of the proposition again.

4. The Selmer group of a semistable elliptic curve. We return to the situation where $k$ is a number field. In the remainder of this paper we will assume that $n$ is an odd prime number. Let $M_{k, 0}$ denote the set of prime ideals of $k$. For any $\alpha \in k^{\times}$let $\Sigma_{k}(\alpha)$ denote the set of prime ideals $\mathfrak{p}$ of $k$ such that $\operatorname{ord}_{\mathfrak{p}}(\alpha) \neq 0$. Let $E$ be a semistable elliptic curve defined over $k$. Thus $\Sigma(E / k):=\Sigma_{k}\left(\Delta_{E}\right)$ is the set of bad prime ideals for $E$. We assume that $E$ has split multiplicative reduction at every prime in $\Sigma(E / k)$. For $\mathfrak{p} \in \Sigma(E / k)$, let $q=q_{\mathfrak{p}}$ be a non-zero element of $k_{\mathfrak{p}}$ with $\operatorname{ord}_{\mathfrak{p}}(q)>0$ such that $E$ is isomorphic to the Tate curve $E_{q} / k_{\mathfrak{p}}$ defined by (3). We fix an isomorphism $\phi_{\mathfrak{p}}: E_{q} \rightarrow E$. We write $\mu_{\mathfrak{p}}, \nu_{\mathfrak{p}}$ and $\tau_{\mathfrak{p}}$ for $\mu, \nu$ and $\tau$ defined in the previous section for $E_{q} / k_{\mathfrak{p}}$. Let

$$
A_{k}=\left\{\mathfrak{p} \in \Sigma(E / k) \mid \nu_{\mathfrak{p}}(S) \neq 0\right\}, \quad B_{k}=\Sigma(E / k) \backslash A_{k} .
$$

Consider the following condition:

$$
\Sigma_{k}(n) \subset \Sigma(E / k) .
$$

Clearly this is equivalent to requiring that $\operatorname{ord}_{\mathfrak{p}}\left(\Delta_{E}\right)>0$ for all $\mathfrak{p} \in \Sigma_{k}(n)$. 
For any subset $X$ of $M_{k, 0}$, we define a subgroup $V(X)$ of $k^{\times} / k^{\times n}$ by

$$
V(X)=\left\{x \in k^{\times} / k^{\times n} \mid \operatorname{ord}_{\mathfrak{p}}(x) \equiv 0(\bmod n)\left(\forall \mathfrak{p} \in M_{k, 0} \backslash X\right)\right\} .
$$

Moreover, if $Y$ is another subset of $M_{k, 0}$ such that $X \cap Y=\emptyset$, we define a subgroup $V(X, Y)$ of $k^{\times} / k^{\times n}$ by

$$
V(X, Y)=\left\{x \in V(X) \mid x=1 \text { in } k_{\mathfrak{p}}^{\times} / k_{\mathfrak{p}}^{\times n}(\forall \mathfrak{p} \in Y)\right\} .
$$

Proposition 4.1. If the condition (11) holds, then

$$
\operatorname{Sel}^{(\psi)}(F / k)=V\left(A_{k}, B_{k}\right) .
$$

Proof. Let $x \in k^{\times} / k^{\times n}$. Then $x$ belongs to $\operatorname{Sel}^{(\psi)}(F / k)$ if and only if $x \in \operatorname{Im}\left(\delta_{\mathfrak{p}}^{(\psi)}\right)$ for all $\mathfrak{p} \in M_{k}$. Since we are assuming that $n$ is odd, it is not necessary to consider the local condition at infinite places. If $\mathfrak{p}$ is a finite place not in $\Sigma(E / k)$ and therefore not dividing $n$, then it is well known that $\operatorname{Im}\left(\delta_{\mathfrak{p}}^{(\psi)}\right)=\mathscr{O}_{\mathfrak{p}}^{\times} / \mathscr{O}_{\mathfrak{p}}^{\times n} \subset k_{\mathfrak{p}}^{\times} / k_{\mathfrak{p}}^{\times n}$, where $\mathscr{O}_{\mathfrak{p}}$ denotes the integer ring of $k_{\mathfrak{p}}$. This shows that $\operatorname{Sel}^{(\psi)}(F / k)$ is a subgroup of $V(\Sigma(E / k))$. If $\mathfrak{p} \in \Sigma(E / k)$, then $E$ has split multiplicative reduction at $\mathfrak{p}$, and so by Proposition 3.1 we have

$$
\operatorname{Im}\left(\delta_{\mathfrak{p}}^{(\psi)}\right)= \begin{cases}k_{\mathfrak{p}}^{\times} / k_{\mathfrak{p}}^{\times n} & \text { if } \mathfrak{p} \in A_{k}, \\ \{1\} & \text { if } \mathfrak{p} \in B_{k} .\end{cases}
$$

Therefore the equality $\operatorname{Sel}^{(\psi)}(F / k)=V\left(A_{k}, B_{k}\right)$ holds.

Corollary 4.2. Assume that the condition (11) holds. If $N \mathfrak{p} \not \equiv 1$ $(\bmod n)$ for all $\mathfrak{p} \in B_{k}$, then

$$
\operatorname{Sel}^{(\psi)}(F / k)=V\left(A_{k}\right) .
$$

Proof. Let $x \in V\left(A_{k}\right)$. Then $\operatorname{res}_{\mathfrak{p}}(x) \in \mathscr{O}_{\mathfrak{p}}^{\times} / \mathscr{O}_{\mathfrak{p}}^{\times n}$ for any $\mathfrak{p} \in B_{k}$. But, since $n$ is a prime number, the assumption that $N \mathfrak{p} \not \equiv 1(\bmod n)$ implies that $\mathscr{O}_{\mathfrak{p}}^{\times n}=\mathscr{O}_{\mathfrak{p}}^{\times}$. Therefore, $x=1$ in $k_{\mathfrak{p}}^{\times} / k_{\mathfrak{p}}^{\times n}$. This proves that $V\left(A_{k}\right) \subset$ $\operatorname{Sel}^{(\psi)}(F / k)$. Thus the assertion follows from Proposition 4.1 .

We will henceforth assume that $k$ contains $\mu_{n}$. For any $\mathfrak{p} \in M_{k, 0} \backslash \Sigma_{k}(n)$ and $x \in k$ with $\operatorname{ord}_{\mathfrak{p}}(x)=0$, let $\left(\frac{x}{\mathfrak{p}}\right)_{n}$ be the $n$th power residue symbol, namely $\left(\frac{x}{\mathfrak{p}}\right)_{n}$ is the $n$th root of unity such that

$$
\left(\frac{x}{\mathfrak{p}}\right)_{n} \equiv x^{(N \mathfrak{p}-1) / n}(\bmod \mathfrak{p})
$$

Note that $\left(\frac{x}{\mathfrak{p}}\right)_{n}=1$ if and only if $x \in \mathscr{O}_{\mathfrak{p}}^{\times n}$. Thus the following corollary immediately follows from Proposition 4.1.

Corollary 4.3. Assume that $k$ contains $\mu_{n}$ and the condition (11) holds. Then

$$
\operatorname{Sel}^{(\psi)}(F / k)=\left\{x \in V\left(A_{k}\right) \mid\left(\frac{x}{\mathfrak{p}}\right)_{n}=1\left(\forall \mathfrak{p} \in B_{k}\right)\right\} .
$$


Now, we will give an explicit description of the set $A_{k}$. For this purpose, divide the set $\Sigma(E / k)$ into two subsets:

$$
\begin{aligned}
& \Sigma^{(1)}(E / k)=\left\{\mathfrak{p} \in \Sigma(E / k) \mid \operatorname{ord}_{\mathfrak{p}}\left(\Delta_{E}\right) \not \equiv 0(\bmod n)\right\} \\
& \Sigma^{(2)}(E / k)=\left\{\mathfrak{p} \in \Sigma(E / k) \mid \operatorname{ord}_{\mathfrak{p}}\left(\Delta_{E}\right) \equiv 0(\bmod n)\right\} .
\end{aligned}
$$

Let $f_{S}$ be a rational function on $E$ satisfying the condition of Proposition 3.1. For any $\mathfrak{p} \in \Sigma(E / k)$ let $f_{\mathfrak{p}, S}$ denote the rational function on $E_{q}$ defined by (6). Since two rational functions $\phi_{\mathfrak{p}}^{*}\left(f_{S}\right)$ and $f_{\mathfrak{p}, S}$ on $E$ have the same divisor, they differ only by non-zero constant multiple:

$$
\phi_{\mathfrak{p}}^{*}\left(f_{S}\right)=c_{\mathfrak{p}} f_{\mathfrak{p}, S} \quad\left(c_{\mathfrak{p}} \in k_{\mathfrak{p}}^{\times}\right) .
$$

But in view of Proposition 2.1 the commutative diagram

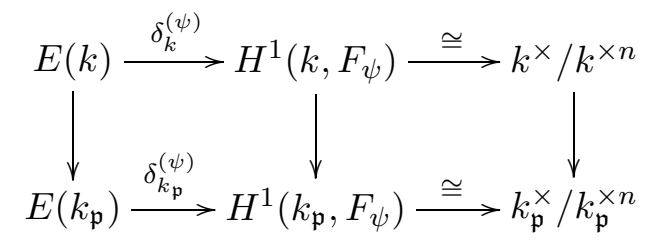

shows that $c_{\mathfrak{p}} \in k_{p}^{\times n}$. Hence, when we compute $\operatorname{Im}\left(\delta_{\mathfrak{p}}^{(\psi)}\right)$, we may use $f_{S}$ instead of $f_{\mathfrak{p}, S}$.

Let $P \in E_{n} \backslash\{O, S\}$. Then the above remark shows that

$$
\operatorname{ord}_{\mathfrak{p}}\left(\phi_{\mathfrak{p}}^{*}\left(f_{S}(P)\right)\right)=\operatorname{ord}_{\mathfrak{p}}\left(f_{\mathfrak{p}, S}\right)
$$

for any $\mathfrak{p} \in \Sigma(E / k)$. For each $\mathfrak{p} \in \Sigma^{(2)}(E / k)$, define the rational number

$$
i_{\mathfrak{p}}(S, P)=\frac{\operatorname{ord}_{\mathfrak{p}}\left(f_{S}(P)\right)}{\frac{1}{n} \operatorname{ord}_{\mathfrak{p}}\left(\Delta_{E}\right)} .
$$

Consider the following condition:

$$
\operatorname{ord}_{\mathfrak{p}}\left(\Delta_{E}\right) \not \equiv 0(\bmod n) \quad \text { for all } \mathfrak{p} \in \Sigma_{k}(n) .
$$

Obviously (12) implies (11).

Proposition 4.4. Assume that $k$ contains $\mu_{n}$ and the condition (12) holds. Then for any prime $\mathfrak{p} \in \Sigma(E / k)$ the following assertions hold:

(i) If $\mathfrak{p} \in \Sigma^{(1)}(E / k)$, then $\nu_{\mathfrak{p}}(S)=0$.

(ii) If $\mathfrak{p} \in \Sigma^{(2)}(E / k) \backslash \Sigma_{k}(n)$ (resp. $\left.\mathfrak{p} \in \Sigma_{k}(n)\right)$, then $i_{\mathfrak{p}}(S, P)$ is an integer (resp. an $n$-adic integer) and the congruence

$$
i_{\mathfrak{p}}(S, P) \equiv-\nu_{\mathfrak{p}}(S) \nu_{\mathfrak{p}}(P)(\bmod n)
$$

holds for any $P \in E(k)_{n} \backslash\{O, S\}$.

Proof. If $\operatorname{ord}_{\mathfrak{p}}\left(\Delta_{E}\right) \not \equiv 0(\bmod n)$, then $q_{1}=q^{1 / n}$ does not belong to $k_{\mathfrak{p}}$. Let $\sigma$ be an element of $\operatorname{Gal}\left(\bar{k}_{\mathfrak{p}} / k_{\mathfrak{p}}\right)$ such that $q_{1}^{\sigma} \neq q_{1}$. Since the Tate 
parametrization $\tau_{\mathfrak{p}}$ is Galois equivariant and $S$ is $k$-rational, we have

$$
\tau_{\mathfrak{p}}\left(\zeta^{\mu_{\mathfrak{p}}(S)} \mathfrak{q}_{1}^{\nu_{\mathfrak{p}}(S)}\right)=\tau_{\mathfrak{p}}\left(\zeta^{\mu_{\mathfrak{p}}(S)}\left(\mathfrak{q}_{1}^{\sigma}\right)^{\nu_{\mathfrak{p}}(S)}\right)
$$

Hence $\nu_{\mathfrak{p}}(S) \tau_{\mathfrak{p}}\left(\mathfrak{q}_{1}^{\sigma-1}\right)=0$. Since $\mathfrak{q}_{1}^{\sigma-1}$ is an $n$th root of unity other than 1 , we have $\tau_{\mathfrak{p}}\left(\mathfrak{q}_{1}^{\sigma-1}\right) \neq 0$. Therefore, $\nu_{\mathfrak{p}}(S)=0$. This proves (i).

To prove (ii), suppose that $\operatorname{ord}_{\mathfrak{p}}\left(\Delta_{E}\right) \equiv 0(\bmod n)$ and $\mathfrak{p}$ does not divide $n$. Then Corollary 3.4 shows that

$$
\frac{\operatorname{ord}_{\mathfrak{p}}\left(f_{S}(P)\right)}{\operatorname{ord}_{\mathfrak{p}}\left(q_{1}\right)} \equiv-\nu_{\mathfrak{p}}(S) \nu_{\mathfrak{p}}(P)(\bmod n) .
$$

Since $\frac{1}{n} \operatorname{ord}_{\mathfrak{p}}\left(\Delta_{E}\right)=\operatorname{ord}_{\mathfrak{p}}\left(q_{1}\right)$, (ii) follows.

Corollary 4.5. Assume that $k$ contains $\mu_{n}$ and the condition (12) holds. Then

$$
A_{k}=\left\{\mathfrak{p} \in \Sigma^{(2)}(E / k) \mid i_{\mathfrak{p}}(S,-S) \not \equiv 0(\bmod n)\right\} .
$$

Proof. By Proposition 4.4(i), $A_{k}$ is a subset of $\Sigma^{(2)}(E / k)$. Let $\mathfrak{p} \in$ $\Sigma^{(2)}(E / k)$. Applying Proposition 4.4(ii) for $P=-S$ and noticing that $\nu_{\mathfrak{p}}(-S) \equiv-\nu_{\mathfrak{p}}(S)(\bmod n)$, we obtain

$$
i_{\mathfrak{p}}(S,-S) \equiv \nu_{\mathfrak{p}}(S)^{2}(\bmod n) .
$$

This implies that $\mathfrak{p} \in A_{k}$ if and only if $i_{\mathfrak{p}}(S,-S) \not \equiv 0(\bmod n)$. The corollary then follows.

5. The Cassels-Tate pairing. We begin with a theorem proved by McCallum [10], which is fundamental in our calculation. It enables us to describe the Cassels-Tate pairing $\langle,\rangle_{\psi}$ defined in (2) in terms of the Hilbert norm residue symbol

of $k_{\mathfrak{p}}$.

$$
(,)_{\mathfrak{p}}: k_{\mathfrak{p}}^{\times} / k_{\mathfrak{p}}^{\times n} \times k_{\mathfrak{p}}^{\times} / k_{\mathfrak{p}}^{\times n} \rightarrow \mu_{n}
$$

TheOREM 5.1. Suppose $E(k)_{n} \cong \mathbb{Z} / n \mathbb{Z} \times \mathbb{Z} / n \mathbb{Z}$ and let $\{S, T\}$ be a basis of $E(k)_{n}$. Let $e_{n}$ denote the Weil pairing on $E_{n}$ and put $\zeta=e_{n}(S, T)$. Let $F=E /\langle S\rangle$ be the cyclic quotient of $E$ by the subgroup $\langle S\rangle$ generated by $S$. Let $x, x^{\prime} \in \operatorname{Sel}^{(\psi)}(F / k)$. For each $\mathfrak{p} \in M_{k}$ let $P_{\mathfrak{p}} \in E\left(k_{\mathfrak{p}}\right)$ be a local point such that $\operatorname{res}_{\mathfrak{p}}(x)=\delta_{\mathfrak{p}}^{(\psi)}\left(P_{\mathfrak{p}}\right)$. Then

$$
\left\langle x, x^{\prime}\right\rangle_{\psi}=\sum_{\mathfrak{p} \in M_{k}} \operatorname{Ind}_{\zeta}\left(f_{T}\left(P_{\mathfrak{p}}\right), x^{\prime}\right)_{\mathfrak{p}},
$$

where $\operatorname{Ind}_{\zeta}: \mu_{n} \rightarrow \mathbb{Z} / n \mathbb{Z}$ denotes the isomorphism sending $\zeta \in \mu_{n}$ to $1 \in$ $\mathbb{Z} / n \mathbb{Z}$ and $f_{T}$ is a rational function on $E$ defined in Proposition 2.1.

Proof. One can prove this in a quite similar way to [10, Theorem 1.4]. See also [2] and [7], where the case $n=5$ is treated. 
The next theorem is proved by Beaver [2] when $n=5$, but the proof works for general $n$. Here we will give a proof based on the result in Section 2 .

THEOREM 5.2. Let the notation and assumption be as in Theorem 5.1. Suppose $E / k$ (and hence $F / k$ ) is a semistable elliptic curve with split multiplicative reduction at every prime in $\Sigma(E / k)$. Let $A_{k}$ be as in Section 3 and assume that the condition (11) holds. For each $\mathfrak{p} \in A_{k}$ put $\lambda_{\mathfrak{p}}=$ $\nu_{\mathfrak{p}}(T) / \nu_{\mathfrak{p}}(S) \in \mathbb{Z} / n \mathbb{Z}$. Then for $x, x^{\prime} \in \operatorname{Sel}^{(\psi)}(F / k)$ we have

$$
\left\langle x, x^{\prime}\right\rangle_{\psi}=\sum_{\mathfrak{p} \in A_{k}} \lambda_{\mathfrak{p}} \operatorname{Ind}_{\zeta}\left(x, x^{\prime}\right)_{\mathfrak{p}}
$$

Proof. Let $\tau_{\mathfrak{p}}: \bar{k}_{\mathfrak{p}}^{\times} / q_{\mathfrak{p}}^{\mathbb{Z}} \rightarrow E\left(\bar{k}_{\mathfrak{p}}\right)$ be the Tate parametrization. For each $\mathfrak{p} \in M_{k}$ there exists a point $P_{\mathfrak{p}} \in E\left(k_{\mathfrak{p}}\right)$ such that $\delta_{\mathfrak{p}}^{(\psi)}\left(P_{\mathfrak{p}}\right)=\operatorname{res}_{\mathfrak{p}}(x)$. Choose $u_{\mathfrak{p}} \in k_{\mathfrak{p}}^{\times}$so that $\tau_{\mathfrak{p}}\left(u_{\mathfrak{p}}\right)=P_{\mathfrak{p}}$. Then by the same argument as in the proof of Proposition 3.1 one can prove that

$$
f_{T}\left(P_{\mathfrak{p}}\right)=f_{T}\left(\tau_{\mathfrak{p}}\left(u_{\mathfrak{p}}\right)\right) \equiv u_{\mathfrak{p}}^{-\nu_{\mathfrak{p}}(T)}\left(\bmod k_{\mathfrak{p}}^{\times n}\right) .
$$

Hence by Theorem 5.1 we have

$$
\left\langle x, x^{\prime}\right\rangle_{\psi}=\sum_{\mathfrak{p} \in M_{k}} \operatorname{Ind}_{\zeta}\left(u_{\mathfrak{p}}^{-\nu_{\mathfrak{p}}(T)}, x^{\prime}\right)_{\mathfrak{p}}
$$

If $\nu_{\mathfrak{p}}(S)=0$, then $\operatorname{Im}\left(\delta_{\mathfrak{p}}^{(\psi)}\right)=\{1\}$ by Proposition 3.1, and so $\left(u_{\mathfrak{p}}^{-\nu_{\mathfrak{p}}(T)}, x^{\prime}\right)_{\mathfrak{p}}=1$. If $\nu_{\mathfrak{p}}(S) \neq 0$, then $u_{\mathfrak{p}}^{-\nu_{\mathfrak{p}}(T)} \equiv\left(u_{\mathfrak{p}}^{-\nu_{\mathfrak{p}}(S)}\right)^{\lambda_{\mathfrak{p}}}\left(\bmod k_{\mathfrak{p}}^{\times n}\right)$. Therefore

$$
\left(u_{\mathfrak{p}}^{-\nu_{\mathfrak{p}}(T)}, x^{\prime}\right)_{\mathfrak{p}}=\left(u_{\mathfrak{p}}^{-\nu_{\mathfrak{p}}(S)}, x^{\prime}\right)_{\mathfrak{p}}^{\lambda_{\mathfrak{p}}}=\left(x, x^{\prime}\right)_{\mathfrak{p}}^{\lambda_{\mathfrak{p}}} \text {. }
$$

The assertion now immediately follows from (13) and (14).

The following theorem shows that one can compute the value of $\lambda_{\mathfrak{p}}$ once the values of the function $f_{S}$ on $E_{n}$ have been known.

TheOREM 5.3. Let the notation and assumption be as in Theorem 5.1. Assume, in addition, that the condition (12) holds. Then for any $\mathfrak{p} \in A_{k}$ the value of $\lambda_{\mathfrak{p}}$ is given by the following formula:

$$
\lambda_{\mathfrak{p}} \equiv-\frac{i_{\mathfrak{p}}(S, T)}{i_{\mathfrak{p}}(S,-S)}(\bmod n) .
$$

Proof. Applying Proposition 4.4(ii) for $P=T$, we obtain

$$
i_{\mathfrak{p}}(S, T) \equiv-\nu_{\mathfrak{p}}(S) \nu_{\mathfrak{p}}(T)(\bmod n) .
$$

Since $i_{\mathfrak{p}}(S,-S) \equiv \nu_{\mathfrak{p}}(S)^{2}(\bmod n)$, it follows that

$$
\frac{i_{\mathfrak{p}}(S, T)}{i_{\mathfrak{p}}(S,-S)} \equiv-\lambda_{\mathfrak{p}}(\bmod n)
$$

which proves the theorem. 
6. The case of $n=3$. Let $E$ be a semistable elliptic curve defined over $\mathbb{Q}$ with a rational point $S$ of order 3 . After a change of coordinates, we may assume that $S=(0,0)$ and $E$ is defined by the Weierstraß equation

$$
y^{2}+a x y+b y=x^{3},
$$

where $a$ and $b$ are integers such that $(a, b)=1$ and $\left(a^{3}-27 b\right) b \neq 0$. The discriminant of $E$ is given by $\Delta_{E}=\left(a^{3}-27 b\right) b^{3}$, and $E$ has split multiplicative reduction at every prime in $\Sigma(E / \mathbb{Q})=\Sigma_{\mathbb{Q}}\left(\left(a^{3}-27 b\right) b\right)$. Let $k$ be a number field containing a cubic root of unity $\zeta$. One can easily see that for any $\mathfrak{p} \in \Sigma(E / k)$ our elliptic curve $E$ considered over $k_{\mathfrak{p}}$ is isomorphic to the Tate curve

$$
E_{q}: \quad y^{2}+x y=x^{3}+\frac{b}{2 a^{3}} x+\frac{b^{2}}{4 a^{6}}
$$

with a non-zero element $q=q_{\mathfrak{p}} \in k_{\mathfrak{p}}$ such that $j\left(E_{q}\right)=j(E)$. The isomorphism $\phi_{\mathfrak{p}}: E \rightarrow E_{q}$ is given by

$$
\phi_{\mathfrak{p}}((x, y))=\left(a^{2} x, a^{3} y-b / 2\right) .
$$

Note that the rational function $y$ on $E$ has the $\operatorname{divisor} \operatorname{div}(y)=3((S)-(O))$. Thus we can take $y$ for the rational function $f_{S}$ on $E$.

Now, let $F=E /\langle S\rangle$ be the quotient of $E$ by the cyclic group generated by $S$ and $\varphi: E \rightarrow F$ the natural surjection. Then $F$ is defined over $\mathbb{Q}$ by

$$
y^{2}+a x y+b y=x^{3}-5 a b x-a^{3} b-7 b^{2} .
$$

Let $\psi: F \rightarrow E$ be the dual isogeny of the isogeny $\varphi$.

Proposition 6.1. Let $k$ be a number field containing $\mu_{3}$ and assume that $\operatorname{ord}_{\mathfrak{p}}(3) \not \equiv 0(\bmod 3)$ for all $\mathfrak{p} \in \Sigma_{k}(3)$. Then $A_{k}=\Sigma_{k}(b)$ and $B_{k}=$ $\Sigma_{k}\left(a^{3}-27 b\right)$. Moreover the $\psi$-Selmer group $\operatorname{Sel}^{(\psi)}(F / k)$ is given by

$$
\operatorname{Sel}^{(\psi)}(F / k)=V\left(\Sigma_{k}(b), \Sigma_{k}\left(a^{3}-27 b\right)\right) .
$$

Proof. The assumption on $k$ ensures that the condition (12) is satisfied. Let $\mathfrak{p} \in \Sigma^{(2)}(E / k)$. Since $f_{S}(-S)=y(-S)=-b$, we have

$$
i_{\mathfrak{p}}(S,-S)=\frac{\operatorname{ord}_{\mathfrak{p}}(b)}{\frac{1}{3} \operatorname{ord}_{\mathfrak{p}}\left(\Delta_{E}\right)} .
$$

It follows that $i_{\mathfrak{p}}(S,-S)=1$ or 0 according as $\mathfrak{p}$ divides $b$ or not. Therefore $A_{k}=V_{k}(b)$ (hence $B_{k}=V_{k}\left(a^{3}-27 b\right)$ ) by Corollary 4.5. Thus the proposition follows from Proposition 4.1.

COROllary 6.2. If every prime factor of $a^{3}-27 b$ is congruent to 2 modulo 3 and $\operatorname{ord}_{3}(b) \not \equiv 0(\bmod 3)$, then

$$
\operatorname{Sel}^{(\psi)}(F / \mathbb{Q})=V\left(\Sigma_{\mathbb{Q}}(b)\right) .
$$

Proof. The assertion follows from Proposition 6.1 and Corollary 4.2. 
Let $K=\mathbb{Q}\left(E_{3}\right)$. Then it is easy to see that $K=\mathbb{Q}\left(\sqrt{-3}, \sqrt[3]{a^{3}-27 b}\right)$. We remark that if $\operatorname{ord}_{3}(b) \not \equiv 0(\bmod 3)$, then $\operatorname{ord}_{\mathfrak{p}}(b) \not \equiv 0(\bmod 3)$ for all $\mathfrak{p} \in \Sigma_{K}(3)$ since the absolute ramification index of $\mathfrak{p}$ is 2 .

Corollary 6.3. Assume that $\operatorname{ord}_{3}(b) \not \equiv 0(\bmod 3)$. Then

$$
\operatorname{Sel}^{(\psi)}(F / K)=\left\{x \in V\left(\Sigma_{K}(b)\right) \mid\left(\frac{x}{\mathfrak{p}}\right)_{3}=1 \text { for all } \mathfrak{p} \in \Sigma_{K}\left(a^{3}-27 b\right)\right\} \text {. }
$$

Proof. The assertion follows from Proposition 6.1 and Corollary 4.3.

Put $\ell=a^{3}-27 b$, hence $K=\mathbb{Q}(\sqrt{-3}, \sqrt[3]{\ell})$. It is not hard to compute all the points of $E_{3}$ explicitly. First, note that

$$
\langle S\rangle=\{O,(0,0),(0,-b)\} .
$$

The coordinates of the points of $E_{3} \backslash\langle S\rangle$ are given as follows:

Lemma 6.4. Let $T$ be a point of order 3 which does not belong to $\langle S\rangle$. Then we have

$$
T=\left(-\frac{(a-\omega \xi)\left(a-\omega^{2} \xi\right)}{9},-\frac{(a-\omega \xi)^{2}\left(a-\omega^{2} \xi\right)}{27}\right),
$$

where $\xi$ is a cubic root of $\ell$ and $\omega$ is a primitive cubic root of unity. (The number of possible choices of the pair $(\xi, \omega)$ is $6=\#\left(E_{3} \backslash\langle S\rangle\right)$.)

Proof. Let $P \in E_{3} \backslash\{O\}$. Then the $x$-coordinate $x(P)$ of $P$ is a root of the quadric equation

$$
3 x^{4}+a^{2} x^{3}+3 a b x^{2}+3 b^{2} x=0 .
$$

The trivial root $x=0$ of this equation corresponds to the points $S=(0,0)$ and $2 S=(0,-b)$. Thus $x(T)$ is a root of the cubic equation

$$
3 x^{3}+a^{2} x^{2}+3 a b x+3 b^{2}=0 .
$$

Solving this equation, we obtain

$$
x(T)=-\frac{3 b}{a-\xi}=-\frac{(a-\omega \xi)\left(a-\omega^{2} \xi\right)}{9}
$$

with some $\xi$ such that $\xi^{3}=\ell$. Here the second equality holds since

$$
27 b=(a-\xi)(a-\omega \xi)\left(a-\omega^{2} \xi\right) .
$$

Solving the quadratic equation $y^{2}+(a x(T)+b) y-x(T)^{3}=0$, we obtain the description of the $y$-coordinate $y(T)$ of $T$ in the lemma.

In the following we fix $\xi$ and consider three (mutually disjoint) subsets $A_{K}^{(i)}(i=0,1,2)$ of $A_{K}$ defined by

$$
A_{K}^{(i)}=\left\{\mathfrak{p} \in A_{K} \mid a \equiv \omega^{i} \xi\left(\bmod \mathfrak{p}^{\varepsilon_{\mathfrak{p}}}\right)\right\},
$$


where $\varepsilon_{\mathfrak{p}}=2$ or 1 according as $\mathfrak{p}$ divides 3 or not. If $b \equiv 0(\bmod 3)$, then

$$
A_{K}=A_{K}^{(0)} \cup A_{K}^{(1)} \cup A_{K}^{(2)}
$$

by (18).

Theorem 6.5. Suppose $\operatorname{ord}_{3}(b) \not \equiv 0(\bmod 3)$. Let $x, x^{\prime} \in \operatorname{Sel}^{(\psi)}(F / K)$. Then

$$
\left\langle x, x^{\prime}\right\rangle_{\psi}=\sum_{\mathfrak{p} \in A_{K}^{(1)}} \operatorname{Ind}_{\zeta}\left(x, x^{\prime}\right)_{\mathfrak{p}}+2 \sum_{\mathfrak{p} \in A_{K}^{(2)}} \operatorname{Ind}_{\zeta}\left(x, x^{\prime}\right)_{\mathfrak{p}},
$$

where $\zeta=e_{3}(S, T)$.

Proof. It follows from Theorem 5.3 that

$$
\lambda_{\mathfrak{p}} \equiv \frac{\operatorname{ord}_{\mathfrak{p}}(y(T))}{\operatorname{ord}_{\mathfrak{p}}(b)}(\bmod 3)
$$

for all $\mathfrak{p} \in A_{K}$. By Lemma 6.4 we have

$$
\operatorname{ord}_{\mathfrak{p}}(y(T))=2 \operatorname{ord}_{\mathfrak{p}}(a-\omega \xi)+\operatorname{ord}_{\mathfrak{p}}\left(a-\omega^{2} \xi\right) .
$$

First, suppose $\mathfrak{p}$ does not divide 3 . Then $\mathfrak{p}$ does not divide simultaneously any two factors of the right hand side of $(18)$. Therefore, if $a \equiv \xi(\bmod \mathfrak{p})$, then $\operatorname{ord}_{\mathfrak{p}}(a-\omega \xi)=\operatorname{ord}_{\mathfrak{p}}\left(a-\omega^{2} \xi\right)=0$. Hence $\operatorname{ord}_{\mathfrak{p}}(y(T))=0$. If $a \equiv \omega \xi$ $(\bmod \mathfrak{p})$, then $\operatorname{ord}_{\mathfrak{p}}(a-\xi)=\operatorname{ord}_{\mathfrak{p}}\left(a-\omega^{2} \xi\right)=0$. Hence

$$
\operatorname{ord}_{\mathfrak{p}}(y(T))=2 \operatorname{ord}_{\mathfrak{p}}(a-\omega \xi)=2 \operatorname{ord}_{\mathfrak{p}}(b) .
$$

Similarly, if $a \equiv \omega^{2} \xi(\bmod \mathfrak{p})$, then $\operatorname{ord}_{\mathfrak{p}}(a-\xi)=\operatorname{ord}_{\mathfrak{p}}(a-\omega \xi)=0$. Hence

$$
\operatorname{ord}_{\mathfrak{p}}(y(T))=\operatorname{ord}_{\mathfrak{p}}(a-\omega \xi)=\operatorname{ord}_{\mathfrak{p}}(b) .
$$

Consequently, if $\mathfrak{p}$ does not divide 3 , then

$$
\operatorname{ord}_{\mathfrak{p}}(y(T))= \begin{cases}0 & \text { if } a \equiv \xi(\bmod \mathfrak{p}), \\ 2 \operatorname{ord}_{\mathfrak{p}}(b) & \text { if } a \equiv \omega \xi(\bmod \mathfrak{p}), \\ \operatorname{ord}_{\mathfrak{p}}(b) & \text { if } a \equiv \omega^{2} \xi(\bmod \mathfrak{p}) .\end{cases}
$$

Next, suppose $\mathfrak{p}$ divides 3 . Then $\operatorname{ord}_{\mathfrak{p}}\left(a-\omega^{i} \xi\right)>0$ for any $i=0,1,2$ and equation (18) shows that

$$
\operatorname{ord}_{\mathfrak{p}}(y(T))=-6+2 \operatorname{ord}_{\mathfrak{p}}(a-\omega \xi)+\operatorname{ord}_{\mathfrak{p}}\left(a-\omega^{2} \xi\right) .
$$

(Note that $\operatorname{ord}_{\mathfrak{p}}(3)=3$.) Moreover, one of the three factors $a-\omega^{i}(i=0,1,2)$ of the right hand side of (18) is divisible by $\mathfrak{p}^{2}$ and the others are not. Therefore, if $a \equiv \xi\left(\bmod \mathfrak{p}^{2}\right)$, then $\operatorname{ord}_{\mathfrak{p}}(a-\omega \xi)=\operatorname{ord}_{\mathfrak{p}}\left(a-\omega^{2} \xi\right)=1$. Hence $\operatorname{ord}_{\mathfrak{p}}(y(T))=-3$. If $a \equiv \omega \xi\left(\bmod \mathfrak{p}^{2}\right)$, then $\operatorname{ord}_{\mathfrak{p}}(a-\xi)=\operatorname{ord}_{\mathfrak{p}}\left(a-\omega^{2} \xi\right)=1$. Hence

$$
\operatorname{ord}_{\mathfrak{p}}(a-\omega \xi)=\operatorname{ord}_{p}\left(\frac{27 b}{(a-\xi)\left(a-\omega^{2} \xi\right)}\right)=\operatorname{ord}_{\mathfrak{p}}(b)+4 .
$$


Therefore,

$$
\operatorname{ord}_{\mathfrak{p}}(y(T))=2\left(\operatorname{ord}_{\mathfrak{p}}(b)+4\right)+1-6=2 \operatorname{ord}_{\mathfrak{p}}(b)+3 .
$$

Similarly, if $a \equiv \omega^{2} \xi\left(\bmod \mathfrak{p}^{2}\right)$, then $\operatorname{ord}_{\mathfrak{p}}\left(a-\omega^{2} \xi\right)=\operatorname{ord}_{\mathfrak{p}}(b)+4$ and $\operatorname{ord}_{\mathfrak{p}}(a-\omega \xi)=1$. Hence

$$
\operatorname{ord}_{\mathfrak{p}}(y(T))=2+\operatorname{ord}_{\mathfrak{p}}(b)+4-6=\operatorname{ord}_{\mathfrak{p}}(b) .
$$

Consequently, if $\mathfrak{p}$ divides 3 , then

$$
\operatorname{ord}_{\mathfrak{p}}(y(T))= \begin{cases}-3 & \text { if } a \equiv \xi\left(\bmod \mathfrak{p}^{2}\right), \\ 2 \operatorname{ord}_{\mathfrak{p}}(b)+3 & \text { if } a \equiv \omega \xi\left(\bmod \mathfrak{p}^{2}\right), \\ \operatorname{ord}_{\mathfrak{p}}(b) & \text { if } a \equiv \omega^{2} \xi\left(\bmod \mathfrak{p}^{2}\right)\end{cases}
$$

By (20) and (21), for any $\mathfrak{p} \in A_{K}^{(i)}(i=0,1,2)$ we have

$$
\operatorname{ord}_{\mathfrak{p}}(y(T)) \equiv-i \cdot \operatorname{ord}_{\mathfrak{p}}(b)(\bmod 3) .
$$

It then follows from (19) that

$$
\lambda_{\mathfrak{p}} \equiv-i(\bmod 3)
$$

for any $\mathfrak{p} \in A_{K}^{(i)}$. Moreover, if $\mathfrak{p} \in M_{K, 0} \backslash A_{K}$, then $\operatorname{ord}_{\mathfrak{p}}(x) \equiv \operatorname{ord}_{\mathfrak{p}}\left(x^{\prime}\right) \equiv 0$ $(\bmod 3)$, and so $\left(x, x^{\prime}\right)_{\mathfrak{p}}=1$. Therefore

$$
\left\langle x, x^{\prime}\right\rangle_{\psi}=\sum_{i=0}^{2} i \sum_{\mathfrak{p} \in A_{K}^{(i)}} \operatorname{Ind}_{\zeta}\left(x, x^{\prime}\right)_{\mathfrak{p}}
$$

This proves the theorem.

7. Proof of Theorem 1.1. We want to show that for a given positive integer $r$ we can find two integers $a$ and $b$ with $(a, b)=1$ and $\left(a^{3}-27 b\right) b \neq 0$ for which

$$
\operatorname{dim}_{\mathbb{Z} / 3 \mathbb{Z}} \amalg\left(F_{(a, b)} / \mathbb{Q}\right)_{3} \geq r .
$$

Let $\ell$ be an odd prime number with $\ell \equiv-1(\bmod 9)$. Thus $\ell$ remains prime in $k:=\mathbb{Q}(\sqrt{-3})$. Let $\xi$ be a cubic root of $\ell$ in $\overline{\mathbb{Q}}$ and put $K=\mathbb{Q}(\sqrt{-3}, \xi)$. Since $\ell \equiv-1(\bmod 9), \ell$ is a cube in $\mathbb{Q}_{3}$, hence $\xi \in \mathbb{Q}_{3}$. Moreover, by genus theory we know that the class number $h$ of $K$ is not divisible by 3 , since the base field $k$ has class number one and $K / k$ is a cyclic extension of degree 3 unramified outside the prime ideal generated by $\ell$.

We choose $r$ prime numbers $p_{1}, \ldots, p_{r}$ with $p_{i} \equiv-1(\bmod 9)$ so that the unique prime ideal of $k$ lying above $p_{i}$ decomposes completely in $K$. This is possible because $\mathbb{Q}\left(\zeta_{9}\right) \cap K=k$. Let

$$
L=k\left(\sqrt[3]{p_{1}}, \ldots, \sqrt[3]{p_{r}}\right) .
$$

Then $L / k$ is a Kummer extension whose Galois group may be described as follows: For each $i$, we can naturally view $\operatorname{Gal}\left(k\left(\sqrt[3]{p_{i}}\right) / k\right)$ as a subgroup of 
$\operatorname{Gal}(L / k)$, and we have an isomorphism

$$
\operatorname{Gal}(L / k) \cong \prod_{i=1}^{r} \operatorname{Gal}\left(k\left(\sqrt[3]{p_{i}}\right) / k\right) .
$$

Choose and fix a primitive cubic root of unity $\omega$, and let $g_{i}$ be the generator of $\operatorname{Gal}\left(k\left(\sqrt[3]{p_{i}}\right) / k\right)$ such that

$$
\sqrt[3]{p_{i}} g_{i}=\omega \sqrt[3]{p_{i}}
$$

Lemma 7.1. There exist prime ideals $\mathfrak{q}_{1}, \ldots, \mathfrak{q}_{r}$ of $K$ such that

$$
\left(\frac{\xi}{\mathfrak{q}_{j}}\right)_{3}=1 \quad \text { and } \quad\left(\frac{p_{i}}{\mathfrak{q}_{j}}\right)_{3}=\omega^{\delta_{i j}}
$$

for all $i, j$, where $\left(\frac{*}{*}\right)_{3}$ denotes the cubic power residue symbol of $K$ and $\delta_{i j}$ denotes Kronecker's delta.

Proof. The extension $K L / k$ is a Kummer extension of exponent 3. Since $\ell$ is relatively prime to $p_{1}, \ldots, p_{r}$, we have an isomorphism

$$
\operatorname{Gal}(K L / k) \cong \operatorname{Gal}(K / k) \times \operatorname{Gal}(L / k) .
$$

Therefore, by Chebotarev's density theorem, there exist prime ideals $\mathfrak{Q}_{1}, \ldots, \mathfrak{Q}_{r}$ of $K L$ such that

$$
\left\{\begin{array}{l}
\left.\operatorname{Frob}_{K L / k}\left(\mathfrak{Q}_{i}\right)\right|_{K}=1, \\
\left.\operatorname{Frob}_{K L / k}\left(\mathfrak{Q}_{i}\right)\right|_{L}=g_{i} .
\end{array}\right.
$$

Let $\mathfrak{q}_{i}$ be the prime ideal of $K$ lying under $\mathfrak{Q}_{i}$. The first condition of (24) implies that $\operatorname{Frob}_{K / k}\left(\mathfrak{q}_{i}\right)=1$ since $\operatorname{Frob}_{K / k}\left(\mathfrak{q}_{i}\right)=\left.\operatorname{Frob}_{K L / k}\left(\mathfrak{Q}_{i}\right)\right|_{K}$. This shows that $\left(\frac{\xi}{\mathfrak{q}_{j}}\right)_{3}=1$. Moreover the second condition of (24) implies that

$$
\sqrt[3]{p_{i}} \operatorname{Frob}_{K L / k}\left(\mathfrak{Q}_{j}\right)=\omega^{\delta_{i j}} \sqrt[3]{p_{i}},
$$

which is equivalent to $\left(\frac{p_{i}}{\mathfrak{q}_{j}}\right)_{3}=\omega^{\delta_{i j}}$. Thus the prime ideals $\mathfrak{q}_{1}, \ldots, \mathfrak{q}_{r}$ have the desired properties.

Letting $\mathfrak{p}_{i}$ and $\mathfrak{q}_{i}$ be as above, choose an integer a such that

$$
\left\{\begin{array}{l}
\operatorname{ord}_{3}(a-\xi)=3, \\
a \equiv \xi\left(\bmod \mathfrak{p}_{1} \ldots \mathfrak{p}_{r}\right), \\
a \equiv \omega \xi\left(\bmod \mathfrak{q}_{1} \ldots \mathfrak{q}_{r}\right) .
\end{array}\right.
$$

The existence of such an integer is ensured by the fact that $\xi \in \mathbb{Q}_{3}$ and the prime ideals $\mathfrak{p}_{i}, \mathfrak{q}_{i}(i=1, \ldots, r)$ decompose completely in $K$ for all $i$. Moreover the first condition of (25) shows that

$$
\Sigma_{K}(3) \subset A_{K}^{(1)}
$$

Since $\operatorname{ord}_{\mathfrak{p}}\left((a-\xi)-\left(a-\omega^{i} \xi\right)\right)=\operatorname{ord}_{\mathfrak{p}}\left(\left(\omega^{i}-1\right)\right)=1$ for any $\mathfrak{p} \in \Sigma_{K}(3)$ and $i=1,2$, this shows that $\operatorname{ord}_{\mathfrak{p}}\left(a-\omega^{i} \xi\right)=1$ for $i=1,2$. Therefore, 
$\operatorname{ord}_{\mathfrak{p}}\left((a-\omega \xi)\left(a-\omega^{2} \xi\right)\right)=2$. In particular, regarding $(a-\omega \xi)\left(a-\omega^{2} \xi\right)$ as an element of $\mathbb{Q}_{3}$, we have $\operatorname{ord}_{3}\left((a-\omega \xi)\left(a-\omega^{2} \xi\right)\right)=1$. Hence the relation

$$
\operatorname{ord}_{3}\left(a^{3}-\ell\right)=\operatorname{ord}_{3}(a-\xi)+\operatorname{ord}_{3}\left((a-\omega \xi)\left(a-\omega^{2} \xi\right)\right)
$$

shows that $\operatorname{ord}_{3}\left(a^{3}-\ell\right)=4$. Therefore, if we put

$$
b=\frac{a^{3}-\ell}{27}
$$

then $b$ is an integer such that $(a, b)=1$ and $\operatorname{ord}_{3}(b)=1$.

Let $E=E_{(a, b)}$ and $F=F_{(a, b)}$ be two elliptic curves defined by the equation in (15) and (17) respectively. Then $K$ coincides with $\mathbb{Q}\left(E_{3}\right)$. Let $S=(0,0) \in E_{3}$ and choose $T \in E_{3} \backslash\langle S\rangle$ so that $e_{3}(S, T)=\omega$, where $\omega$ is the primitive cubic root of unity defined in (23). We claim that

$$
\operatorname{dim}_{\mathbb{Z} / 3 \mathbb{Z}}\left(H(F / \mathbb{Q})_{\psi}\right) \geq r .
$$

Since $\amalg(F / \mathbb{Q})_{\psi} \subset \amalg(F / \mathbb{Q})_{3}$, this proves the claim (22). To prove (26), let $\beta_{j}$ be a generator of the principal ideal $\mathfrak{q}_{j}^{h}$ for each $j=1, \ldots, r$. (Recall that $h$ is the class number of $K$.) Before proving (26) itself, we prove a lemma.

LEMMA 7.2. Let the notation be as above. Then $p_{1}, \ldots, p_{r} \in \operatorname{Sel}^{(\psi)}(F / \mathbb{Q})$ and $\beta_{1}, \ldots, \beta_{r} \in \mathrm{Sel}^{(\psi)}(F / K)$.

Proof. Since $\ell=a^{3}-27 b$ is a prime number with $\ell \equiv 2(\bmod 3)$, Corollary 6.2 shows that

$$
\operatorname{Sel}^{(\psi)}(F / \mathbb{Q})=V\left(\Sigma_{\mathbb{Q}}(b)\right) .
$$

In particular, $p_{1}, \ldots, p_{r} \in \operatorname{Sel}^{(\psi)}(F / \mathbb{Q})$.

To prove the second statement, notice that $K \supset \mu_{3}$. Let $\mathfrak{l}=(\xi)$ denote the unique prime ideal in $K$ lying above $\ell$. Then by Corollary 6.3 we have

$$
\operatorname{Sel}^{(\psi)}(F / K)=\left\{x \in V\left(\Sigma_{K}(b)\right) \mid\left(\frac{x}{\mathfrak{l}}\right)_{3}=1\right\} .
$$

Thus, in order to prove that $\beta_{i} \in \operatorname{Sel}^{(\psi)}(F / K)$, we have to show that $\left(\frac{\beta_{i}}{\mathfrak{l}}\right)_{3}=1$. But this is equivalent to $\left(\xi, \beta_{i}\right)_{\mathfrak{l}}=1$. To compute $\left(\xi, \beta_{i}\right)_{\mathfrak{l}}$, note that

$$
\left(\xi, \beta_{i}\right)_{\mathfrak{q}_{i}}=\left(\frac{\xi}{\mathfrak{q}_{i}}\right)_{3}^{h}=1 .
$$

The first equality holds because $\operatorname{ord}_{\mathfrak{q}_{i}}(\xi)=0$ and $\operatorname{ord}_{\mathfrak{q}_{i}}\left(\beta_{i}\right)=h$, and the second one holds by Lemma 7.1. Moreover, since $\xi \equiv-1(\bmod 9)$, we have $\left(\xi, \beta_{i}\right)_{\mathfrak{p}}=1$ for all $\mathfrak{p} \in \Sigma_{K}(3)$. Then the product formula implies that $\left(\xi, \beta_{i}\right)_{\mathfrak{r}}=1$. This proves that $\beta_{i} \in \operatorname{Sel}^{(\psi)}(F / K)$, completing the proof.

We return to the proof of (26). For this, it suffices to show that the images of $p_{1}, \ldots, p_{r} \in \operatorname{Sel}^{(\psi)}(F / \mathbb{Q})$ in $H(F / \mathbb{Q})_{\psi}$ are linearly independent. Since we have a homomorphism $\operatorname{Sel}^{(\psi)}(F / \mathbb{Q}) \rightarrow \operatorname{Sel}^{(\psi)}(F / K)$ induced from 
the natural map $\mathbb{Q}^{\times} / \mathbb{Q}^{\times 3} \rightarrow K^{\times} / K^{\times 3}$, it is enough to show that the images of $p_{1}, \ldots, p_{r} \in \operatorname{Sel}^{(\psi)}(F / K)$ in $H(F / K)_{\psi}$ are linearly independent. For this purpose, we calculate the Cassels-Tate pairing $\left\langle p_{i}, \beta_{j}\right\rangle_{\psi}$ on $\operatorname{Sel}^{(\psi)}(E / K)$ for all $i, j$.

We first note that $\left(p_{i}, \beta_{j}\right)_{\mathfrak{p}}=1$ for all $\mathfrak{p} \in \Sigma_{K}(3)$ because $p_{i} \equiv-1$ $(\bmod 9)$. For each $i$ there are three conjugate ideals of $\mathfrak{p}_{i}$ in $K$. We number them so that

$$
\Sigma_{K}\left(p_{i}\right) \cap A_{K}^{(\nu)}=\left\{\mathfrak{p}_{i}^{(\nu)}\right\} \quad(\nu=0,1,2) .
$$

Thus $\mathfrak{p}_{i}^{(0)}=\mathfrak{p}_{i}$. Moreover, by the choice of the integer $a$ in (25) we have

$$
\left\{\begin{array}{l}
\Sigma_{K}\left(\beta_{i}\right) \cap A_{K}^{(1)}=\left\{\mathfrak{q}_{i}\right\}, \\
\Sigma_{K}\left(\beta_{i}\right) \cap A_{K}^{(\nu)}=\emptyset \quad(\nu=0,2) .
\end{array}\right.
$$

Therefore, applying Theorem 6.5 , we obtain

$$
\left\langle p_{i}, \beta_{j}\right\rangle_{\psi}=\operatorname{Ind}_{\omega}\left(p_{i}, \beta_{j}\right)_{\mathfrak{p}_{i}^{(1)}}+2 \operatorname{Ind}_{\omega}\left(p_{i}, \beta_{j}\right)_{\mathfrak{p}_{i}^{(2)}}+\operatorname{Ind}_{\omega}\left(p_{i}, \beta_{j}\right)_{\mathfrak{q}_{j}}
$$

Since $p_{i}$ is in $k$, we have

$$
\left(p_{i}, \beta_{j}\right)_{\mathfrak{p}_{i}^{(1)}}=\left(p_{i}, \beta_{j}\right)_{\mathfrak{p}_{i}^{(2)}}=\left(p_{i}, N_{K / k}\left(\beta_{j}\right)\right)_{p_{i}} .
$$

Hence the sum of the first two terms of the right hand side of (27) is equal to zero. On the other hand, we have

$$
\left(p_{i}, \beta_{j}\right)_{\mathfrak{q}_{j}}=\left(\frac{p_{i}}{\mathfrak{q}_{j}}\right)_{3}^{h}=\omega^{h \delta_{i j}}
$$

by Lemma 7.1. Consequently, we obtain the following simple description of the pairing $\left\langle p_{i}, \beta_{j}\right\rangle_{\psi}$ :

$$
\left\langle p_{i}, \beta_{j}\right\rangle_{\psi} \equiv h \delta_{i j}(\bmod 3) .
$$

Since $h$ is not divisible by 3 , the equality $\left\langle p_{i}, \beta_{j}\right\rangle_{\psi}=0$ holds if and only if $i \neq j$, which proves that $p_{1}, \ldots, p_{r}$ are independent in $\operatorname{Sel}^{(\psi)}(E / K)$. This proves (26), completing the proof of Theorem 1.1.

\section{References}

[1] D. Atake, On elliptic curves with large Tate-Shafarevich groups, J. Number Theory 87 (2001), 282-300.

[2] C. D. Beaver, 5-torsion in the Shafarevich-Tate group of a family of elliptic curves, ibid. 82 (2000), 25-46.

[3] V. R. Bölling, Die Ordnung der Schafarewitsch-Tate-Gruppe kann beliebig groß werden, Math. Nachr. 67 (1975), 157-179.

[4] A. Brumer and K. Kramer, The rank of elliptic curves, Duke Math. J. 44 (1977), 715-743.

[5] J. W. S. Cassels, Arithmetic on curves of genus 1. IV. Proof of the Hauptvermutung, J. Reine Angew. Math. 211 (1962), 95-112. 
[6] J. W. S. Cassels, Arithmetic on curves of genus 1. VI. The Tate-Shafarevich group can be arbitrarily large, ibid. 214 (1963), 65-70.

[7] T. Fisher, On 5 and 7 descents for elliptic curves, $\mathrm{PhD}$ thesis, Cambridge, 2000.

[8] K. Kramer, A family of semistable elliptic curves with large Tate-Shafarevich groups, Proc. Amer. Math. Soc. 89 (1983), 379-386.

[9] F. Lemmermeyer, On Tate-Shafarevich groups of some elliptic curves, in: Algebraic Number Theory and Diophantine Analysis (Graz, 1998), F. Halter-Koch and R. F. Tichy (eds.), de Gruyter, 2000, 277-291.

[10] W. G. McCallum, On the Shafarevich-Tate group of the Jacobian of a quotient of the Fermat curve, Invent. Math. 93 (1988), 637-666.

[11] S. Milne, Arithmetic Duality Theorems, Perspect. Math. 1, Academic Press, 1986.

[12] P. Roquette, Analytic Theory of Elliptic Functions over Local Fields, Hamburger Math. Einzelschriften (N.F.) 1, Vandenhoeck \& Ruprecht, Göttingen, 1970.

[13] J. H. Silverman, The Arithmetic of Elliptic Curves, Grad. Texts in Math. 106, Springer, 1986.

[14] - Advanced Topics in the Arithmetic of Elliptic Curves, Grad. Texts in Math. 151, Springer, 1994.

[15] J. Tate, Duality theorems in Galois cohomology over number fields, in: Proc. Internat. Congr. Mathematicians (Stockholm, 1962), Inst. Mittag-Leffler, Djursholm, 1963, 288-295.

Department of Mathematics

Rikkyo University

Nishi-Ikebukuro, Toshima-ku

Tokyo 171-8501, Japan

E-mail: aoki@rkmath.rikkyo.ac.jp

Received on 11.2.2002

and in revised form on 14.2.2003 